\section{Las TICS como factor clave en la gestión académica y administrativa de la universidad}

\author{
TICS as a key factor in the academic and administrative \\ management of the university
}

\section{RESUMEN}

En el contexto de las universidades, actualmente hay coincidencias en reconocer, la influencia de las tecnologías, en la gestión universitaria; así como del impacto y desafío que tiene que hacer frente a los nuevos retos de la gestión académica y administrativa.

El estudio de investigación concentrará su perspectiva en la dimensión del desarrollo tecnológico, este es un fenómeno de rápida acumulación de información de conocimiento. Las nuevas tecnologías de información y de las telecomunicaciones ofrecen un potencial enorme de transformación de los sistemas educativos y de difusión de sus aplicaciones productivas; que se logra mediante una actividad sistemática de usos de las capacidades de la mente, (conocida como conocimiento e investigación) lo que hace crucial que su implementación y desarrollo académico demande una gestión administrativa con modelos organizativos, que impliquen calidad para que sus procesos y recursos interactúen con el uso intensivo de la tecnología. Las tecnologías podrían permitir el desenvolvimiento de sistemas de aprendizaje paraleles al sistema educativo formal que ahora conocemos, estos poseerían sus propios títulos y certificaciones; con flexibilidad institucional y adaptada a las necesidades específicas de cada educando. El rol de la gestión administrativa, en la implementación y desarrollo de las tecnologías, demanda sustituir sus modelos organizativos asumiendo una gestión de calidad acreditada, mejora contínua y que garantice la excelencia y la competitividad.

Palabras claves: Universidad; Tecnología; Información; Comunicaciones; Gestión del Conocimiento; Gestión académica; gestión administrativa.

\footnotetext{
ABSTRACT

Now a days there are coincidences in recognizing that in the context of universities the influence of the technologies in university management is gravitating, as well as the impact and challenge that has to face the new challenges of the academic and administrative management of the university .

The research study will concentrate its perspective on the dimension of technological development, it is a phenomenon of rapid accumulation of information of knowledge. The new information technologies and telecommunications offer an enormous potential for transforming educational systems and disseminating their productive applications, which is achieved through a systematic activity of uses of the capacities of the mind, known as knowledge and research that makes it crucial that its aca-
}

Manuel Jorge Espinoza Altamirano

manuelespinoza123@yahoo.es

Universidad Nacional Mayor de San Marcos, Facultad de Ciencias Administrativas

(C) Los autores. Este artículo es publicado por Gestión en el Tercer Milenio de la Facultad de Ciencias Administrativas de la Universidad Nacional Mayor de San Marcos. Este es un artículo de acceso abierto, distribuido bajo los términos de la licencia Creative Commons Atribucion - No Comercia_Compartir Igual 4.0 Internacional. (http://creativecommons.org/licenses/by-nc-sa/4.0/) que permite el uso no comercial, distribución y reproducción en cualquier medio, siempre que la obra original sea debidamente citada. 
demic implementation and development request administrative management with organizational models that imply quality in its processes and resources interact with the intensive use of technology. The technologies allow the development of learning systems parallel to the formal education system we now know, systems with their own titles and certifications, institutional flexibility and adapted to the specific needs of each student. The role of the administrative administration in the implementation and development of technologies demands replacing its organizational models assuming an accredited quality management, continuous improvement and guaranteeing excellence and competitiveness.

Keywords: University; Technology; Information; Communications; Knowledge Management; Academic Management; Administrative Management.

\section{INTRODUCCIÓN}

En este periodo post moderno del siglo XXI, ha habido cambios científicos, tecnológicos, económicos, sociales y políticos en un entorno global e hipercompetitivo que impone la necesidad de reformular y actualizar el sistema de gestión tecnológica y administrativa; el cual hace viable el enfrentar los nuevos desafíos de un sistema educativo superior frente a los modelos de calidad, mejora continua, competitividad y excelencia.

Dentro de esos avances, se tiene como factor relevante el desarrollo del conocimiento, la tecnología de la información y comunicaciones; permitiendo una gestión académica y administrativa más eficaz en las universidades, como factor esencial. Según Sales (2003)“Una organización que se introduce en el tema de la mejora continua y la gestión tecnológica define una estructura organizativa para este efecto".

De esta manera, se inicia con la concepción de una visión, el punto de partida para desarrollar una cultura tecnológica. Actualmente, la nueva ley universitaria 30220 promueve la investigación y el desarrollo tecnológico en las universidades del país. En la actualidad, la Universidad Mayor de San Marcos suscribió un convenio en el 2017FONDECEYT CONCYTEC para la implementación del Sistema Integrado de Gestión (SIG) con el objetivo de mejorar los procesos y actividades en las diferentes dependencias de la universidad. Según Castellano (2003) "los esfuerzos para adoptar la gestión tecnológica son inútiles, si la alta gerencia de la organización no está comprometida con la organización". Para lograr este cometido, debe transferirse la visión de futuro hacia todos los niveles de la institución; por lo que se hace necesario definir la misión, políticas educativas, plataforma tecnológica, sistemas y programas de calidad.

\section{OBJETIVO}

Contribuir a los procesos de modernización considerando el uso de las tecnologías de la información y comunicaciones como sistemas flexibles para el desarrollo de las actividades académicas, de investigación y de gestión administrativa en la Universidad.

\section{MÉTODOS}

Para el desarrollo del presente estudio se utilizó el tipo de investigación con enfoque cualitativo y el método de análisis y síntesis. El diseño de investigación es no experimental, que estudia los fenómenos para después analizar y no requiere de pruebas; y, finalmente la aplicativa para la utilización del conocimiento existente.

\section{Contexto mundial y Latinoamericano de las TICS}

De acuerdo al World Economic Forum, el Perú se mantiene en el puesto 90 en el ranking, según la XIV edición del Informe Global de Tecnología de la Información 2015, reporte que evalúa el impacto de las TICs en el proceso de desarrollo y competitividad de 143 economías del mundo. El reporte muestra que está aumentando la brecha digital entre naciones y lo cual debe ser "motivo de gran preocupación, dado el ritmo implacable del desarrollo tecnológico. Las naciones menos desarrolladas corren el riesgo de quedar más atrasadas y se necesitan urgentes acciones concretas para abordarlo. 
Tabla 1

Ranking Mundial (Top 10)del impacto de las TICS

\begin{tabular}{|c|c|c|c|}
\hline Economia/Pais & 2015 & 2014 & Variación \\
\hline Singapur & 1 & 2 & $\Rightarrow$ \\
\hline Finlandia & 2 & 1 & $\checkmark$ \\
\hline Suecia & 3 & 3 & $\Rightarrow$ \\
\hline Holanda & 4 & 4 & $\Rightarrow$ \\
\hline Noruega & 5 & 5 & $\Rightarrow$ \\
\hline Suiza & 6 & 6 & $\Rightarrow$ \\
\hline Estados Unidos & 7 & 7 & $\Rightarrow$ \\
\hline Reino Unido & 8 & 9 & 순 \\
\hline Luxemburgo & 9 & 11 & 순 \\
\hline Japón & 10 & 16 & 담 \\
\hline
\end{tabular}

Fuente: Foro Económico Mundial (WEF), 2015

Tabla 2

Ranking Latinoamericano del impacto de las TICS

\begin{tabular}{|lcccc}
\hline \multicolumn{1}{c}{ Economia/Pais } & 2015 & 2014 & Puntaje & Variación \\
\hline Chile & 38 & 35 & 4.6 & \\
\hline Uruguay & 46 & 56 & 4.5 & \\
\hline Costa Rica & 49 & 53 & 4.4 & \\
\hline Panamá & 51 & 43 & 4.4 & \\
\hline Colombia & 64 & 63 & 4.1 & \\
\hline México & 69 & 79 & 4 & \\
\hline El Salvador & 80 & 98 & 3.9 & \\
\hline Brasil & 84 & 69 & 3.9 & \\
\hline Perú & 90 & 90 & 3.7 & \\
\hline Argentina & 91 & 100 & 3.7 & \\
\hline República Dominicana & 95 & 93 & 3.6 & \\
\hline Venezuela & 103 & 106 & 3.4 & \\
\hline Paraguay & 105 & 102 & 3.4 & \\
\hline Bolivia & 111 & 120 & 3.3 & \\
\hline Nicaragua & 128 & 124 & 2.9 & \\
\hline Haiti & 137 & 143 & 2.5 & \\
\hline
\end{tabular}

Fuente: Foro Económico Mundial (WEF), 2015.

Singapur (1) y Finlandia (2) encabezan el ranking en la clasificación del 2015, como resultado del aprovechamiento de tecnologías de información y comunicaciones como factores que impulsan a la competitividad. El informe identifica varios países que hicieron mejoras considerables, tanto en términos de su índice de puntuación como en su clasificación. Perú se mantiene en la misma posición que el año 2014. Su desarrollo se ve limitado por barreras tales como el sistema educativo de baja calidad (Puesto 133), baja calidad de la educación en matemáticas y ciencias (138), que dificultan la preparación del país para hacer un buen uso de las tecnologías de la información, así como la falta de eficacia de los órganos legislativos (140), que retrasan el entorno regulatorio para las TIC. En Latinoamérica, los países que figuran 2015 en la mitad superior de la tabla son los siguientes: Chile (38), Uruguay (46), Costa Rica (49), Panamá (51), Colombia (64), y México (69) y por detrás se encuentra Perú (90), que se mantiene en la misma posición por encima de Argentina (91), Republica Dominicana (95), Venezuela (103), Paraguay (105), Bolivia (111).

\section{Estructura de Gestión de las TICS en las Universidades}

Es importante contar con rutas claras sobre las herramientas facilitadoras que vayan más allá de solo vincularlas a las posibles soluciones a temas de costos, incremento de acceso y calidad en el contexto de los procesos educativos. Por ello, lo que hace necesario plantear esquemas integradores que representen, de manera completa, todos los niveles de innovación, sus antecedentes y desarrollo. Así como el alcance de su influencia en los diversos miembros de la comunidad universitaria.

Por su parte, Sahraoui (2009) señala que las universidades, (especialmente aquellas de países en vías de desarrollo), su principal dificultad en la gestión de las TIC: la carencia de una planificación estratégica con enfoque prospectivoy que oriente este proceso.

Así mismo, este autor plantea empezar creando una estructura sostenible de gobierno de las TIC, siendo flexibles ante las diversas modalidades de aprendizaje basado en recursos abiertos; conciliar las perspectivas académicas, administrativas y técnico-financieras; integrar las TIC con la misión formativa de la institución y construir una cultura digital a nivel de la comunidad universitaria.

Por su parte, Al-Mobaideen (2009) destaca ciertos factores críticos a tomar en cuenta si es que se desea gobernar la integración exitosa de las TIC en la institución educativa, a saber: estrategias, políticas, infraestructura, redes, financiación, sostenibilidad, y cultura institucional influenciada por orientaciones internas y externas en el ámbito de la educación superior. Según dicho autor, una estrategia de alcance de las TICS, (que involucra estos factores), posibilitan que las universidades 
puedan contar con una planificación más flexible; establezcan mejor su grupo objetivo, fortalezcan sus procesos de administración y estén más comprometido con la enseñanza y el aprendizaje (p. 7).

El modelo propuesto por Al-Mobaideen (2009) busca ser una propuesta integradora de los diferentes niveles institucionales que requiere la integración de las TIC. Por ello, presenta una estructura de tres niveles y destaca en cada uno de ellos, los elementos claves a tener en cuenta, tal como se presenta en la figura $\mathrm{N}^{\circ} 1$ :
La primera sección de este modelo integrador, correspondiente al macro-sistema, considera el importante rol que tienen las universidades de estar alineadas con los cambios del entorno. En este sentido, se incluyen la competencia, la globalización, las relaciones con pares y las nuevas tecnologías; factores críticos para el desarrollo e implementación de las TIC. Asimismo, en un marco socioeconómico, esta aproximación identifica las prioridades, retos, fortalezas, debilidades, oportunidades y amenazas que tiene la institución respecto a la integración de las TIC; según su misión y objetivos (Al-Mobaideen,

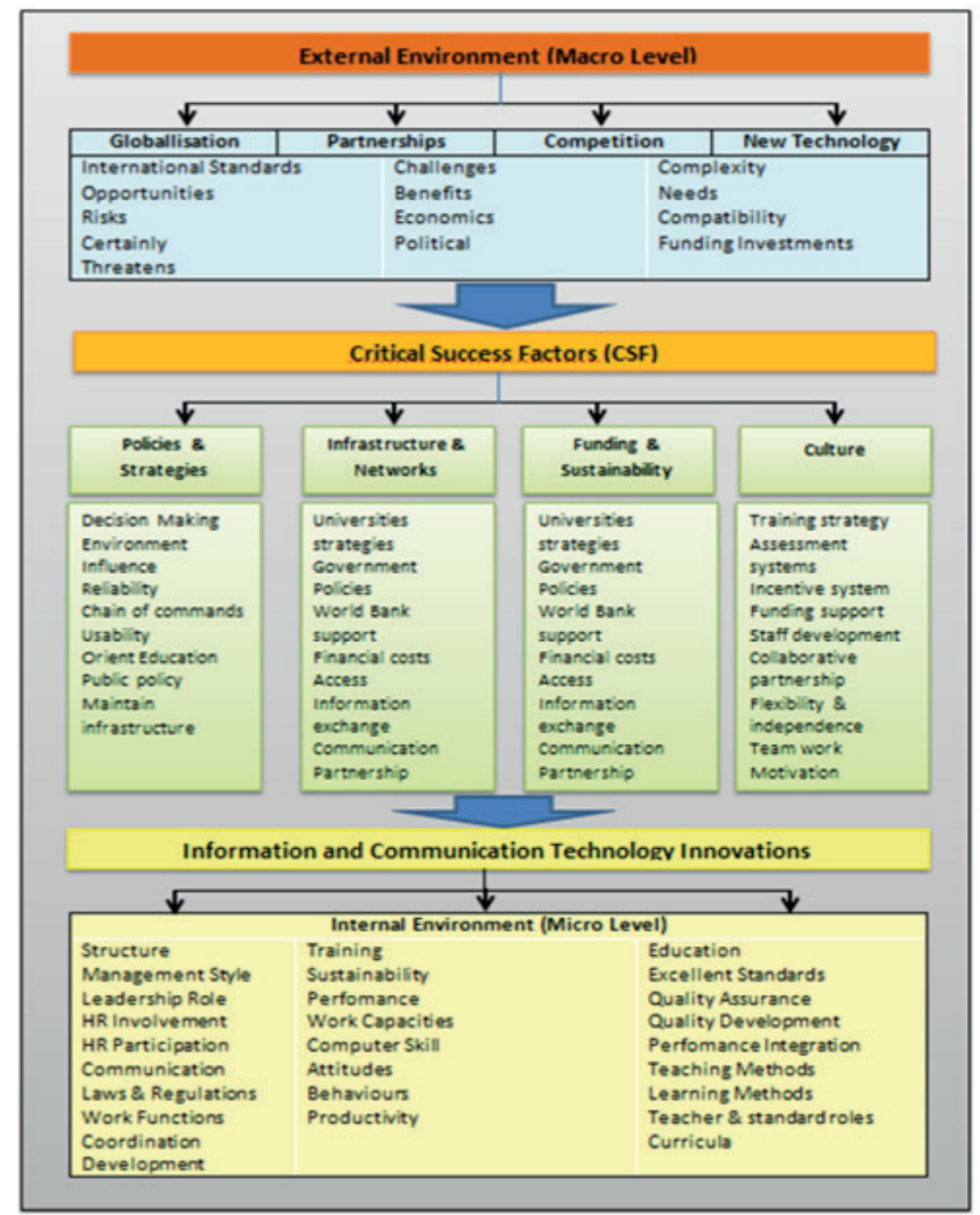

Figura 1. Factores críticos para la integración exitosa de las TIC en las Universidades.

Fuente: Al-Mobaideen (2009) 
2009). La segunda sección corresponde a los factores críticos para obtener éxito, los cuales se encuentran estrechamente ligados a las características del entorno (macrosistema). Estos factores representan los componentes más importantes para realizar una óptima integración de las TIC, pues justifican la estrategia de incorporación de estas tecnologías en el ámbito universitario y definen la toma de decisiones sobre este tema (elaboración de políticas, planificación de los recursos de financiación, gestión de las innovaciones en la cultura institucional). En resumen, tener en cuenta estas variables permite incrementar la eficiencia de las funciones y procesos internos de la universidad al momento de adoptar las TIC (Al-Mobaideen, 2009). La tercera sección, correspondiente al micro-sistema, incluye el sistema propio de las instituciones de educación superior y las actividades llevadas a cabo frecuentemente. Por ello, se consideran temas vinculados a la pedagogía, el currículo, los procesos de enseñanza-aprendizaje; así como las características de los actores educativos y miembros de la comunidad universitaria. A su vez, esta sección se ve directamente influenciada por el macro-sistema y los factores críticos (Al-Mobaideen, 2009). El planteamiento de este modelo se sustenta en la importancia de una óptima gobernanza de las TIC en las instituciones de educación superior, la cual se sitúa en el contexto del cambio hacia la cultura digital. En palabras de Uceda (2013), la mayor parte de las universidades se ha centrado en lograr una administración eficiente de los recursos tecnológicos; mas no basta con concebir a las TIC como un elemento táctico gestionado de manera vertical y aislada, sino que deben tener carácter estratégico y estar alineadas con los objetivos globales o corporativos de la institución.

\section{Retos de la Universidad Nacional Mayor De San Marcos}

La Universidad Nacional Mayor de San Marcos, así como otras instituciones y empresas, una buena parte de ellas, en el siglo XXI asumieron, buena parte de ellas, una estructura organizativa formal inscrita en el modelo de organización burocrático, y aun en la actualidad coexisten con otros modelos organizativos técnicamente denominados modelos tradicionales, acompañados de una gestión clásica tradicional. Los significativos cambios económicos, sociales, políticos y tecnológicos producidos en las últimas décadas en el mundo; han generado que los modelos organizativos y de gestión con mejores cualidades, hayan sido superados técnicamente por otros modelos modernos flexibles, penetrados por los avances tecnológicos y que pueden hacer frente con mayor eficiencia y eficacia, productividad, calidad y competitividad a los nuevos desafíos de los entornos local, sectorial, nacional y global.

La Universidad Nacional Mayor de San Marcos, con miras a que el 2021 esté insertado en las Tecnología de la Información y Comunicación (TIC), deberá asumir en un entorno másdinámico; en la que las tendencias de las mutaciones en la ciencia, tecnología, innovación; así como los cambios políticos, económicos, sociales, ecológicos del Perú estarán insertados con más fuerza en el entorno global. Esto servirá de estímulo para generar, en la universidad en forma intensiva, un rol de gran importancia y de trascendencia como un centro académico y de investigación; por renovadas estructuras organizacionales por procesos y por redes capaces de producir conocimientos, ciencia y tecnologías con estándares competitivos internacionales sostenibles; en materias vinculadas a suministrar el soporte multidisciplinarios suficiente para el desarrollo económico, social, cultural y de responsabilidad social en el ámbito nacional y con reconocimiento global.

\section{Gestión del conocimiento}

Del Moral (2007) señala que la gestión del conocimiento consiste en poner a disposición del conjunto de miembros de una institución de un modo ordenado, práctico y eficaz, además de los conocimientos explicitados. Se asume, con esta afirmación, que los conocimientos particulares son sobreentendidos (tácitos); que pueda ser útiles para el más inteligente y funcionamiento de la misma y el máximo desarrollo y crecimiento de dicha institución. Ello implica:

1. Una concepción del trabajo en equipo que equilibre individualismos $\mathrm{y}$ cooperación, y que elimine competencia 
entre los miembros de la institución para proyectarla al exterior.

2. Considerar los conocimientos como algo fundamental, imprescindible y rentable. Qué signifique eficacia.

3. Ser consciente de que los conocimientos que disponen las instituciones, y los lideres, implica relaciones de causaefecto de los que su miembros no son conscientes por completo.

4. Abrir la puerta a la investigación sobre descubrimientos de conocimientos, de extrema utilidad para la institución, aún desconocidos.

Peluffo \& Catalán, (2002) señala que la principal tarea de la gestión del conocimiento, es manejar, administrar y aprovechar esta avalancha de información y para ello debe formar y reorganizar el capital intelectual, de las personas que trabajan, por ejemplo, en una organización con el propósito de desarrollar la "capacidad de aprender y generar conocimiento nuevo o mejorar el que existe". Este concepto también puede ser aplicado a la gestión de los procesos que generan información dentro de las organizaciones o instituciones. Para su uso provechoso se identifican cinco dimensiones:

a) Un proceso de producción del conocimiento por medio de aprendizajes organizacionales.

b) Un espacio de conocimiento, que puede ser una región, una ciudad, una organización.

c) La presencia de herramientas y tecnologías degestión del conocimiento para documentar el conocimiento organizacional.

d) Una sinergia resultante del proceso de desarrollo de un sistema, la capacidad de respuestas de las comunidades frente a lo nuevo en un medio inestable y cambiante.

e) La presencia de trabajadores del conocimiento.

Así mismo, señala que la aparición de la gestión del conocimiento fue en respuesta a la incapacidad de las prácticas gerenciales tradicionales para administrar eficientemente el conocimiento tácito y su transformación a explícito. "Si este no se gestiona de alguna forma, ya sea almacenándolo, haciéndolo circularo gestionando las competencias de quienes participan en esa realidad; se corre el riesgo de perder el principal factor diferenciador que promueve la sinergia en la innovación o en el cambio". En ese sentido, afirma que el objetivo de la gestión de conocimiento apunta a "generar, compartir y utilizar el conocimiento tácito (know-how) y explícito (formal) existente en una determinado espacio; para dar respuestas a las necesidades de los individuos y de las comunidades en su desarrollo". Paluffo y Catalán, (202) sostienen que este proceso debe dar los siguientes resultados:

a) Poner en funcionamiento los medios necesarios para conseguir la información y el conocimiento que precisa una persona, una comunidad o región en el momento oportuno; por medio de herramientas para analizar la información y fortalecer la capacidad de responder a las ideas que se obtienen, a partir de esa información y del conocimiento tácito que estos poseen.

b) Administrar el conocimiento organizacional y el aprendizaje organizacional con el fin de fortalecer la institucionalidad que va a implantar estrategias de desarrollo de mediano y largo plazo.

c) Construir marcos (procesos) integrados más eficientes a partir de la construcción de modelos de futuros, cuyo soporte será el conocimiento estratégico que le dará eficacia y seguridad al proceso.

d) Crear una base tecnológica adecuada al contexto y espacio donde se va a aplicar, por la cual circule el conocimiento como el caso de las redes universitarias con la economía; conectar las diversas regiones aprovechando las experiencias más exitosas y las formas en que fueron superados o solucionados los 
errores más frecuentes. Esto permite solucionar con mayor velocidad los problemas y adaptarse con más flexibilidad.

"La apropiación del conocimiento se da por medio del aprendizaje, por lo que la capacidad de aumentar el stock de conocimiento va a depender en parte por la capacidad que tenga la persona o la organización de aprender", dicen Paluffo y Catalán (202). Agregan que la renovación del conocimiento origina a su vez la innovación, que constituye un elemento sustancial para la generación de nuevos conocimientos. En ese contexto, quien quiera iniciar un proceso de gestión de conocimiento debe identificar el conocimiento tácito de las personas a fin de promover "un proceso continuo de innovación".

El modelo educativo de la UNMSM define a la gestión de conocimiento como el conjunto de estrategias de generación, divulgando y aplicación de los conocimientos que contribuyen a los procesos fundamentales de la universidad y generan mayores posibilidades de desarrollo personal, institucional y social.

En ese sentido, la gestión del conocimiento de la universidad se entiende como la capacidad para generar conocimiento, divulgarlos y aplicarlos en beneficio delahumanidadygenerar ideas innovadoras e innovar continuamente. Promover el desarrollo y gestión del talento humano, aplicando los conocimientos para desarrollar productos y servicios.

Según Nonaka y Takeuchi (1995), una organización que crea conocimiento es capaz de generar conocimiento nuevo, internalizarlo e introducirlo dentro de un proceso de innovación exitoso. Es la principal generadora de procesos de innovación por medio de una espiral permanente de producción de nuevas dimensiones, a partir del conocimiento nuevo que se va incorporando a sus procesos, productos, servicios y sistema.

\section{Gestión de las TICS}

Según Pérez \& Dressler, (2007) la especial importancia en la evolución de las TIC tiene el desarrollo del software, que ha permitido la aparición de avanzadas herramientas informáticas de gestión; con nuevas funcionalidades y aplicaciones empresariales, entre las que se pueden destacar las siguientes:

- Intranets: Red privada de una organización diseñada y desarrollada siguiendo los protocolos propios y el funcionamiento de Internet, protocolo TCP/IP, navegador web, etc. Su utilización es interna pero puede estar conectada a Internet y a otras redes externas. Para los usuarios se resume en una serie de páginas Web que dan acceso a la distinta documentación de la empresa, informaciones corporativas, aplicaciones informáticas; incluso permiten la publicación de información y conocimientos personales de cada empleado. Además, dentro de las Intranet se pueden organizar y tener acceso a comunidades de prácticas virtuales, foros y listas de distribución.

- Software de Simulación y realidad virtual: aplicaciones que permiten minimizar los costes de la realización de prototipos, experimentar nuevas ideas y simular la aplicación de conocimientos.

- Workflow: aplicaciones que permiten, mediante herramientas informáticas, automatizar las fases que componen la elaboración de un proceso de negocio. Facilita la distribución, seguimiento y ejecución de las tareas o flujos que componen un trabajo; indicando en qué fase se encuentra el trabajo, quién es el encargado de la ejecución de cada fase, qué procedimientos se tienen que seguir y qué incidencias suceden durante las mismas.

- Videoconferencias:Sistemaquepermite a varias personas, con independencia de su ubicación geográfica, entablar mediante aplicaciones específicas una conversación con soporte audio y video prácticamente en tiempo real.

- Datamining: tecnología que permite la explotación y análisis de los datos almacenados por la organización, generalmente una gran cantidad de datos almacenados en bases de 
datos y datawarehouse, buscando entre ellos relaciones y patrones de comportamiento no observables directamente.

- Datawarehouse: Repositorio o almacén de datos de gran capacidad que sirve de base común a toda la organización. Almacena los datos procedentes tanto del interior de la organización como del exterior organizándolos por temas, lo que facilita su posterior explotación.

- Inteligencia artificial: Aplicaciones informáticas a las que se dota de propiedades asociadas a la inteligencia humana. Ejemplos son los sistemas expertos, redes neuronales, etc. que a partir del conocimiento y reglas introducidas por un experto humano permiten alcanzar inferencia y resolver problemas.

- Motores de búsqueda: software diseñado para rastrear fuentes de datos tales como bases de datos, Internet, etc; lo que permite indexar su contenido y facilitar su búsqueda y recuperación.

- Gestión documental: Aplicaciones que permiten la digitalización de documentos, su almacenamiento, el control de versiones y su disponibilidad para los usuarios con autorización para su consulta y/o modificación.

- Mapas de conocimiento y páginas amarillas: Directorios que facilitan la localización del conocimiento dentro de la organización mediante el desarrollo de guías y listados de personas, o documentos, por áreas de actividad o materias de dominio.

- Mensajería instantánea y correo electrónico: aplicaciones que facilitan la comunicación en tiempo real o diferido, así como el intercambio de documentos.

- Groupware: Tecnologías diseñadas para la gestión de trabajos en equipo. Facilita coordinar el trabajo y compartirinformacionesy aplicaciones informáticas.

El premio nobel de física Arno Allan Penzias escribió en 1989: "La tecnología ofrece basta posibilidades, pero solo si se tiene la imaginación suficiente para aprovecharlas". El enfoque tecnológico por el contrario trata acerca que la información tecnológica debiera proporcionarse como soporte para la gestión del conocimiento. Existe en la actualidad un conjunto de herramientas y tecnología de la información idóneas para la gestión del conocimiento. Del Moral (2007).

\section{MODELO EDUCATIVO}

La Universidad Nacional Mayor de San Marcos ha formulado un modelo educativo planteado en el año 2015 con la finalidad de dar a conocer a la comunidad académica y a la sociedad en general los argumentos filosóficos, científicos, culturales y pedagógicos que sirven de sustento a la formación universitaria; lo que exige un pensamiento divergente, crítico y autocritico y creativo capaz de dar soluciones diferentes e innovadoras a los problemas científicos, sociales y políticos; desafío que la universidad tiene que afrontar y dar solución. El objetivo de la Universidad es la formación integral de profesionales, la producción y difusión del conocimiento a través de la investigación humanística, científica y tecnológica, contribuyendo al país con responsabilidad social.

El modelo educativo que se ofrece a la sociedad se asienta en tres columnas:

1. La realidad socio educativa del país, a la cual responde la universidad.

2. Las megatendencias y tendencias educativas internacionales

3. La fundamentaciones pedagógicos realizada mediante la recopilación y síntesis de distintas teorías y enfoques.

El enfoque de formación por competencias es uno de los desafíos de la educación superior en el mundo (Unesco 1998) por tal razón, varios países latinoamericanos están trabajando en esa perspectiva educativa. 
En la sociedad del conocimiento, la investigación es parte fundamental del desarrollo sostenido y racional de la sociedad; debiendo insertarnos en un renovado modelo de organización por procesos y asumir la gestión pertinente.

Una gestión al servicio del ciudadano necesariamente deberá cambiar el tradicional modelo de organización funcional y migrar hacia una organización por procesos contenidos en las "cadenas de valor" de cada entidad, que aseguren que los bienes y servicios públicos de sus responsabilidad generen resultados e impactos positivos para el ciudadano, dados los recursos disponibles. Los procesos son definidos como una secuencia de actividades que transforman una entrada o insumo (un solicitud de un bien o un servicio) en una salida (la entrega del bien o el servicio), añadiéndole un valor en cada etapa de la cadena (mejores condiciones de calidad/ precio, rapidez, facilidad, comodidad, entre otros), (PNMGP, 2013).

\section{INTERPRETACIÓN DE DATOS O DISCUSIÓN}

Existe actualmente la percepción de que la tecnología de información y comunicación constituye un factor predominante en el desarrollo académico y administrativos de las Universidades, proporcionando mayores competencias en la formación profesional y eficacia en los procesos administrativos. Estas TICS comprenden el avance de las computadoras, software, internet, la telefonía, realidad virtual y las aplicaciones multimedia. Las tecnologías de la información y comunicaciones están constituidas por los procesos de creación de la información, almacenamiento de la información, transmisión de la información generación y administración de los sistemas información. Las TICS constituyen un factor clave en la gestión, en el mejoramiento continuo y éxito de estas Instituciones. En efecto, la gestión tecnológica, dentro de un marco administrativo eficaz, permite una apropiada interacción entre la tecnología, el recurso humano y el conocimiento generado y asimilado; lo que conlleva a un mejoramiento continuo en la calidad de los bienes o servicios ofrecidos tanto a nivel de productividad y de competitividad. Cabe puntualizar que la generación, la asimilación y el uso efectivo de la tecnología mediante la gestión tecnológica, incluye también la utilización eficiente y eficaz de todos los recursos asignados por la organización.

La investigación, constituye el eje fundamental en el desarrollo académico de la universidad y se encuentra amparado en la nueva Ley Universitaria 30220. El uso de las TICS como soporte y herramienta en las actividades y proyectos de investigación facilitan el desarrollo y la producción sistemática del conocimiento y la tecnología, cuyos resultados favorecen y aportan a la comunidad universitaria, a la sociedad y otros grupos de interés en este mundo de alta competitividad y globalización.

La educación universitaria peruana se inicia en la UNMSM y ha sufrido procesos de cambio a lo largo de la historia. En la sociedad actual, la abundancia y la disponibilidad de información, la complejidad del conocimiento y la velocidad de los cambios tienen como efecto la obsolescencia de los conocimientos adquiridos y hábitos de enseñanza-aprendizaje. Los métodos tradicionales de formación profesional utilizados en la universidad peruana, no permiten atender, en forma satisfactoria, los nuevos retos de una educación superior de calidad, debido a la rigidez de los currículos y los programas de estudio, a la desactualización de la normatividad institucional, la aislamiento en que se encuentran respecto a la sociedad, y a las estrategias pedagógicas convencionales utilizadas en los procesos de aprendizajeenseñanza.

Según UNESCO, la formación profesional son "Todas las formas y niveles del proceso educativo que incluyenademás del conocimiento general, el estudio de las tecnologías y de las ciencias relacionadas, la adquisición de habilidades prácticas, de competencias, actitudes y comprensiones relacionadas con las ocupaciones en los diferentes sectores de la vida social".

\section{CONCLUSIONES}

Las nuevas tecnologías de información y las comunicaciones ofrecen un potencial enorme de transformación de los sistemas educativos y de difusión de sus aplicaciones productivas, que 
se logra mediante una actividad sistemática de usos de las capacidades de la mente; conocida como conocimiento e investigación, lo que hace crucial que su implementación y desarrollo académico demande una gestión administrativa eficaz con modelos organizativos, que impliquen calidad en el que sus procesos y recursos interactúen con el uso intensivo de la tecnología.

La implementación de la gestión por procesos, en el marco de una gestión orientada a resultados, constituye un elemento central de un sistema de gestión moderno; creando o agregando valor para todos los estamentos de la universidad, contribuyendo así lograr los resultados esperados.

La gestión del conocimiento en las universidades es precisamente la creación de grupos interdisciplinarios orientados a desarrollar actividades y proyectos de investigación, teniendo como soporte las TICs y para dinamizar, viabilizar y gestionar estas actividades y proyectos debe incluirse dentro de un sistema integrado de gestión.

\section{RECOMENDACIONES}

Se recomienda utilizar y aprovechar los beneficios de las nuevas tecnologías de información y de las telecomunicaciones ya que nos ofrece un potencial enorme de transformación de los sistemas educativos. Estos permitirían lograr un mayor desarrollo del conocimiento e investigación en las ciencias y la tecnología, siendo crucial para el desarrollo académico. Del mismo modo, contribuiría a la gestión eficaz en la administración de la universidad.

Desarrollar una investigación de impacto que esté dirigida a fortalecer los procesos de adaptación y apropiación, de Tecnologías de Información y Comunicaciones, que ofrecen a sus estudiantes, egresados, docentes y personal administrativo.

El manejo de conceptos como tecnología, innovación y transferencia tecnológica favorece una mayor apropiación y asimilación de estos por parte de las universidades grandes; es decir, aquellas que tiene sede central; observándose que, independientemente del tamaño de la universidades, el tratamiento estratégico de temas tecnológicos compete directamente a la rectoría y, en el mejor de los casos, a los Consejos Superiores y las decisiones las apoyan con los conceptos de planeación o departamentos de sistemas.

\section{REFERENCIAS BIBLIOGRÁFICAS}

Al-Mobaideen, H. (2009). ICT diffusion in JordanianUniversities. European and MediterraneanConference on Information Systems, Izmir, Turquía.

Castellano, R. (2003). La tecnología en la gerencia de empresa. Mexico. Editorial Garza.

Cuesta, L.O. (2000). La gestión tecnológica como avance científico. Madrid. Arabit, Editores, S.A.

Del Moral, Anselmo (2007). La gestión del conocimiento. Madrid. Thomson editores.

Hernandez, Roberto (2010) Metodologia de la investigación. Mexico. Mc Graw Hill Interamericana Editores.

NakanoOsores (2014). "Integración y Gobernanza de las TIC en las Universidades: análisis situacional de la PUCP". Tesis para optar el grado de Magíster Política y Gestión Universitaria. Lima: Universidad Catolica Del Perú.

NONAKA, I. y TAKEUCHI, H. (1995): The knowledgecreating company: How Japanese Companies Create the Dynamics of Innovation. Oxford UniversityPress. New York-Oxford.

M. Peluffo, E. Catalán: Introducción a la gestión del conocimiento y su aplicación al sector público. Instituto Latinoamericano y del Caribe de Planificación Pérez, Daniel y Dressler, Michael (2007).“Tecnologías de la información para la gestión del conocimiento". Económica y SocialILPES, Serie de Manuales no 22. (2002)

Pérez, Daniely Dressler, Michael (2007).“Tecnologías de la información para la gestión del conocimiento". Intangible Capital. Vol. 3. Madrid. España.

Sales, D.O. (2003). La gestión tecnológica. México. Editorial Jalisco.

Uceda, J. (2013). Estrategias y gobierno TI en la universidad española. Madrid: Universidad Politécnica de Madrid.

Revista Modelo Educativo San Marcos-UNMSM 2015

Sahraoui H (2009) scholar.google.es/ citations?user $=x s U k T C E A A A A J \& h l=e s$ 